\title{
The $(\alpha, n)$ reaction cross-section calculations for some isotopes of iron group elements in the range of 5-20 MeV
}

\author{
Ercan Yildiz* \\ Kırıkkale University, Department of Physics, Kırıkkale, Turkey
}

\begin{abstract}
Iron group elements ( $\mathrm{Sc}, \mathrm{Mn}, \mathrm{Fe}, \mathrm{Co}, \mathrm{Ni}$ ) are especially very important because structural material selection in design of fusion-fission reactors is very crucial. Obtained results from the nuclear reactions using structural materials can be used developing for these structural materials some isotopes. For this reason, in this study the cross sections of ${ }^{45} \mathrm{Sc}(\alpha, n){ }^{48} \mathrm{~V},{ }^{55} \mathrm{Mn}(\alpha, \mathrm{n}){ }^{58} \mathrm{Co},{ }^{54} \mathrm{Fe}(\alpha, n){ }^{57} \mathrm{Ni},{ }^{59} \mathrm{Co}(\alpha, \mathrm{n}){ }^{62} \mathrm{Cu}$, ${ }^{62} \mathrm{Ni}(\alpha, n){ }^{65} \mathrm{Zn}$ reactions have been calculated at $5-20 \mathrm{MeV}$ energy ranges by using TALYS 1.8 and NONSMOKER codes. Obtained results were compared experimental data from EXFOR database.
\end{abstract}

\section{Introduction}

Nuclear fusion is a safe and clean energy source which serves unlimited energy to mankind. One of the most important advantages of a fusion energy system is the abundant fusion fuel availability in the nature, contrary to relatively scarce fission fuel resources. Furthermore, a fusion energy system has aspects of an attractive product with respect to safety and environmental advantages compared to other energy sources. Thus, market penetration of fusion energy reactors may create a revolution in energy generation in near future. Nevertheless, the market penetration of merchant pure fusion reactors have not been expected before the year 2050. On the other hand, the combination of a fusion and fission reactor may have realistic chances for a relatively earlier introduction of fusion power plants for electricity production [1].

The first wall, blanket system is the structural core materials for nuclear fusion reactors. The success of nuclear fusion reactors is highly dependent on performance and reliability of the first wall, blanket system which is the core structural materials for fusion reactors. Therefore, the selection of structural materials is extremely important for fusion reactor technology [25]. These materials can be classified their activation characteristics. Sc, $\mathrm{Mn}$, and Fe are considered as low activation elements while elements such as Nickel (Ni), Cobalt $(\mathrm{Co})$ are some of the high activation materials [6].

The knowledge of nuclear data in the fusion technology has a great importance in the domains of nuclear structure, nuclear decay, and nuclear reaction. The experimental measurements model theory calculations and systematic predictions are rather useful to provide this knowledge, especially for predicting the cross-sections of nuclear reaction [7].

In this study, cross sections of the ${ }^{45} \mathrm{Sc}(\alpha, n){ }^{48} \mathrm{~V}$, ${ }^{55} \mathrm{Mn}(\alpha, \mathrm{n}){ }^{58} \mathrm{Co}, \quad{ }^{54} \mathrm{Fe}(\alpha, \mathrm{n}){ }^{57} \mathrm{Ni}, \quad{ }^{59} \mathrm{Co}(\alpha, \mathrm{n}){ }^{62} \mathrm{Cu}$, ${ }^{62} \mathrm{Ni}(\alpha, \mathrm{n})^{65} \mathrm{Zn}$ reactions have been calculated with the TALYS 1.8 [8] and NON-SMOKER [9] codes. Obtained results were compared with experimental data from EXFOR [10]

\section{Results and Conclusions}

The $(\alpha, n)$ reactions cross sections of given reactions were calculated in the incident alpha energy range of 5 to $20 \mathrm{MeV}$ by using default values in the TALYS 1.8 and NON-SMOKER codes. We compared to the calculated data and experimental data from EXFOR database in Figs. 1-5.

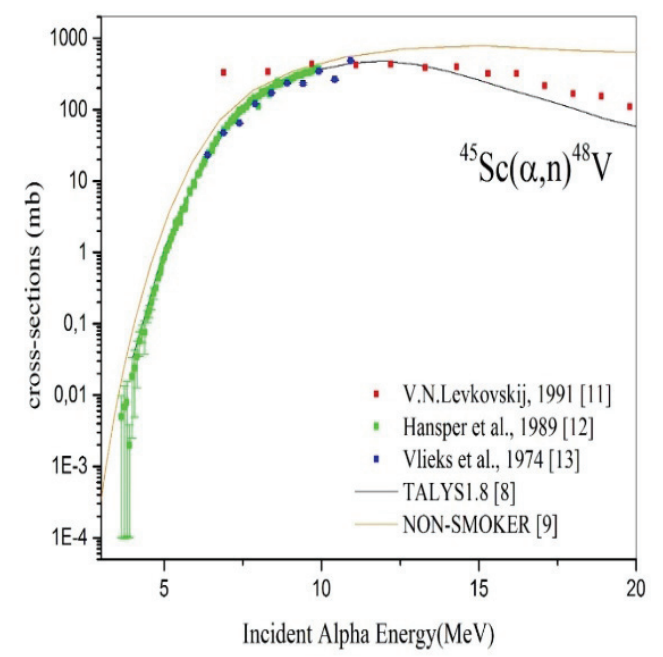

Fig. 1. Comparison of experimental and evaluated crosssections of ${ }^{45} \mathrm{Sc}(\alpha, n){ }^{48} \mathrm{~V}$ as a function $\alpha$ energy. 


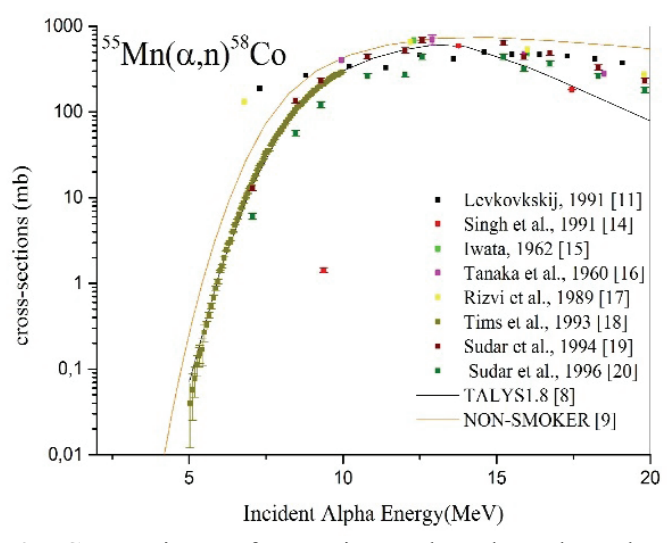

Fig. 2. Comparison of experimental and evaluated crosssections of ${ }^{55} \mathrm{Mn}(\alpha, n){ }^{58} \mathrm{Co}$ as a function $\alpha$ energy.

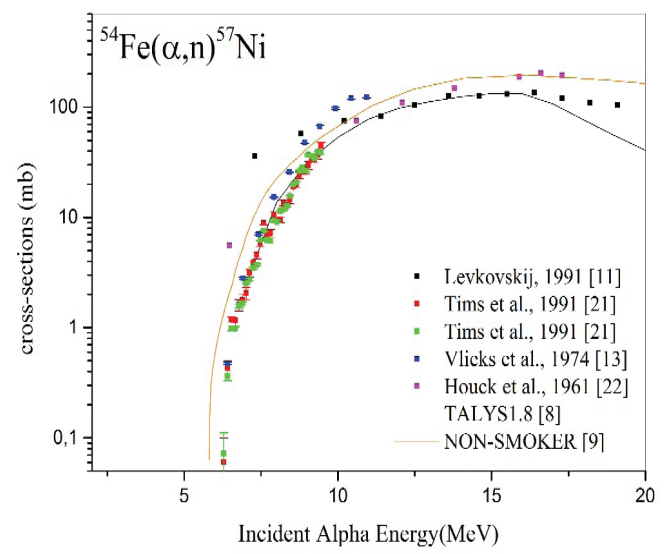

Fig. 3. Comparison of experimental and evaluated crosssections of ${ }^{54} \mathrm{Fe}(\alpha, \mathrm{n})^{57} \mathrm{Ni}$ as a function $\alpha$ energy.

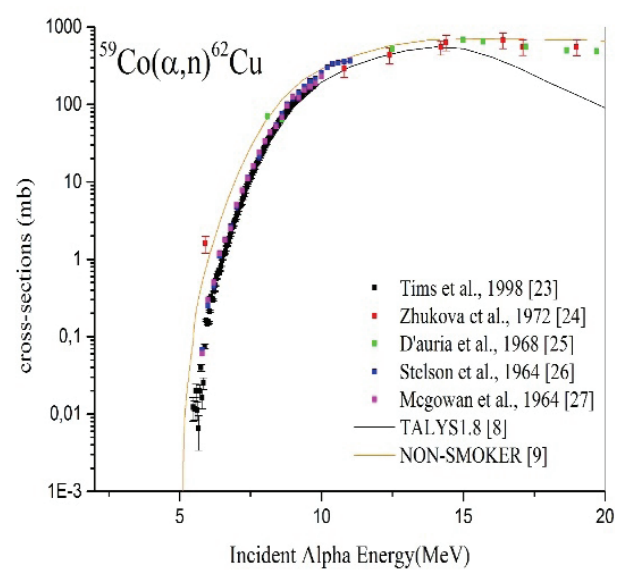

Fig. 4. Comparison of experimental and evaluated crosssections of ${ }^{59} \mathrm{Co}(\alpha, n){ }^{62} \mathrm{Cu}$ as a function $\alpha$ energy.

We can see that the calculated data by TALYS 1.8 are agrement with all the experimental data for ${ }^{45} \mathrm{Sc}(\alpha, n)^{48} \mathrm{~V}$ reaction.

It can be seen that the calculated cross-section data by TALYS 1.8 are agrement with the experimental data at low energies for ${ }^{55} \mathrm{Mn}(\alpha, \mathrm{n}){ }^{58} \mathrm{Co},{ }^{54} \mathrm{Fe}(\alpha, n){ }^{57} \mathrm{Ni}$, ${ }^{59} \mathrm{Co}(\alpha, n){ }^{62} \mathrm{Cu},{ }^{62} \mathrm{Ni}(\alpha, n){ }^{65} \mathrm{Zn}$ reaction at low energies especially.

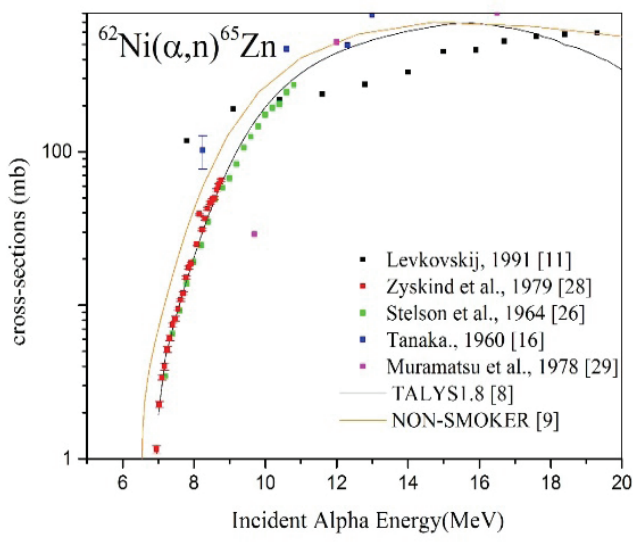

Fig. 5. Comparison of experimental and evaluated crosssections of ${ }^{62} \mathrm{Ni}(\alpha, n)^{65} \mathrm{Zn}$ as a function $\alpha$ energy.

\section{References}

[1] M. Ubeyli, Materials and Design 28 (2007)

[2] W.R. Meier et al., Lawrence livermore national laboratory,LLNLJRNL-416976 (2009)

[3] IAEA Publication, Development of radiation resistant reactor core structural materials,http://www.iaea.org/About/Policy/ GC/GC51/GC51InfDocuments/English/gc51inf-3-att7 en.pdf

[4] M. Rubel, Trans Fusion Sci Technol 53 (2008)

[5] M. Victoria et al., Nucl. Fusion 41, 8 (2001)

[6] P.M. Raole et al., Trans. IIM 62 (2009)

[7] E. Tel et al., J. Fusion Energy 35, (2016)

[8] A. J. Koning, H. Hilaire, S. Goriely, TALYS-1.8, NRG, Netherland, http://www.talys.eu (2017)

[9] http://nucastro.org/nonsmoker.html

[10] https://www-nds.iaea.org/exfor/exfor.htm

[11] V.N. Levkovskij, Act.Cs.by Moscow (1991)

[12] V.Y.Hansper et al., Nuclear Physics,551 (1993)

[13] A.E.Vlieks et al., Nuclear Physics, 224 (1974)

[14] B.P. Singh et al., Canadian Journal of Physics Vol.69 (1991)

[15] S.J. Iwata, Journal of the Physical Society of Japan Vol.17 (1962)

[16] S.Tanaka, Journal of the Physical Society of Japan Vol.15 (1960)

[17] I.A.Rizvi, et al., Canadian Journal of Physics Vol.67 (1989)

[18] S.G. Tims, Nuclear Physics, Section A Vol.563 (1993)

[19] S .Sudar, et al., Phys. Rev, Part C, Nuclear Physics Vol.50 (1994)

[20] S .Sudar, et al., Phys. Rev, Part C, Nuclear Physics Vol.53 (1996)

[21] S.G. Tims, Nuclear Physics, Section A Vol.524 (1991)

[22] F.S.Houck, et al., Phys. Rev Vol.123 (1961)

[23] S.G. Tims, Nuclear Physics, Section A Vol.483 (1998)

[24] O.A. Zhukova, et al.,Fizika Vol.16 (1972)

[25] J.M. D’auria, et al., Phys. Rev Vol.168 (1968)

[26] P.H. Stelson, et al.,Phys. Rev.133 (1964)

[27] F.K. Mcgowan, et al., Phys. Rev.133 (1964)

[28] J.L. Zyskind, et al., Nuclear Physics, Section A Vol.331 (1979)

[29] H. Muramatsu, et al., Applied Radiation and Isotopes Vol.29 (1978) 